\title{
Frontiers in Numerical Optimization of Heat Sinks
}

\author{
Gerardo Maria Mauro, PhD \\ Department of Engineering, Università degli Studi del Sannio, Benevento (Italy), Piazza Roma 21
}

\begin{abstract}
We live the age of revolutions that will change the World, named energy \& digital transition and Industry 4.0. One key aspect of such revolutions lies in micro- and nano-technologies, i.e., in the capillary diffusion of miniaturized electronic devices in industrial, IT equipment and automotive sectors. The high thermal power "produced" by these systems, coupled with the lack of well-designed heat sinks, can cause system failure and permanent damages. Since the chances of that happening increase with the system miniaturization and complexity, the mentioned revolutions amplify the need of optimizing the thermal design of heat sinks, focusing on electronics' cooling devices. Moreover, the great progresses in additive manufacturing and 3D printing techniques are strongly changing the general approach to the problem formulation. Here, the focus is on the latest explored ways and frontiers in the numerical optimization of heat sinks' thermal performance, e.g., shape, size and topology optimizations, arranging the main design approaches and revealing the main limitations, e.g., geometrical, computational, and unsolved issues, together with conventional and innovative manufacturing processes. Therefore, ranging from active - i.e., smart surfaces - to passive - i.e., optimizing heating surfaces - heat sinks' enhancement, the aim is to summarize the guidelines for an integrated design procedure.
\end{abstract}

